\title{
A Chargeless Complex Vector Matter Field in Supersymmetric Scenario
}

\author{
L. P. Colatto ${ }^{1}$ and A. L. A. Penna ${ }^{2,3}$ \\ ${ }^{1}$ CEFET/RJ UnED Petrópolis, 25620-003 Petrópolis, RJ, Brazil \\ ${ }^{2}$ Instituto de Física, University of Brasília, Brasília, DF, Brazil \\ ${ }^{3}$ International Center for Condensed Matter Physics, University of Brasília, CP 04513, 70919-970 Brasília, DF, Brazil
}

Correspondence should be addressed to L. P. Colatto; lcolatto@gmail.com

Received 25 April 2015; Revised 25 June 2015; Accepted 26 July 2015

Academic Editor: Shaaban Khalil

Copyright (C) 2015 L. P. Colatto and A. L. A. Penna. This is an open access article distributed under the Creative Commons Attribution License, which permits unrestricted use, distribution, and reproduction in any medium, provided the original work is properly cited. The publication of this article was funded by SCOAP ${ }^{3}$.

\begin{abstract}
We construct and study a formulation of a chargeless complex vector matter field in a supersymmetric framework. To this aim we combine two nochiral scalar superfields in order to take the vector component field to build the chargeless complex vector superpartner where the respective field strength transforms into matter fields by a global $U(1)$ gauge symmetry. For the aim of dealing with consistent terms without breaking the global $U(1)$ symmetry we imposes a choice to the complex combination revealing a kind of symmetry between the choices and eliminates the extra degrees of freedom which is consistent with the supersymmetry. As the usual case the mass supersymmetric sector contributes as a complement to dynamics of the model. We obtain the equations of motion of the Proca's type field for the chiral spinor fields and for the scalar field on the mass-shell which show the same mass as expected. This work establishes the first steps to extend the analysis of charged massive vector field in a supersymmetric scenario.
\end{abstract}

\section{Introduction}

Matter field dynamics was firstly established by Dirac in a consistent relativistic framework. He has studied the free electron dynamics where its interaction yields the first steps on QED, which was further developed by Feynmann and others $[1,2]$. These studies were very important for the formulation and the understanding of QFT, standard model, and also the strings theory. Indeed it has been the basement of all theoretical analysis of any dynamics which is concerned with integer or half-integer spin particles. In fundamental quantum theory we have classified into two types: boson and fermions, respectively. Fermions usually are the constituent of the matter and bosons are the interaction particle [3]. Nevertheless if we are treating to the weak force we have to deal with charged (or not) massive vector (boson) fields which are the intermediate between the protons and neutrons. So we could interpret as charged vector matter fields.
Due to the supersymmetry which plays a fundamental role on strings theory fitting together quantum theories of the gravitational interactions, electroweak and strong forces, the studies on supersymmetric theories are of great interest to high energy physicists such as applications in particle physics [4] and supersymmetry breaking [5] and in the treatment of classical supergravity [6] with inclusion of topological ChernSimons terms [7]. Furthermore, supersymmetry is required to understand the thermodynamics of quantum gravity [8], to build new scenarios for the electroweak baryogenesis in high energies $[9,10]$ as well as establishing superstring theories correctly [11-16]. Supersymmetry deals with graded Lie algebra in the unique reliable algebra extension which holds to be consistent with the S-matrix in relativistic quantum field theory [17-20]. Recalling that this special symmetry correlates fermionic and bosonic fields, called superpartners, which puts them together in a superfield formulation. It remarks the important role played by the study of matter-like 
vector fields to construct appropriated supersymmetric models $[21,22]$. Moreover supersymmetric models with chiral superfields and global gauge invariance involving matter fields are elegantly constructed $[16,20,22]$. Thus quarks, leptons, and vector bosons which participate in usual gauge theories, as electroweak theory and chromodynamics, in a supersymmetric extension coexist along their superpartners: squarks, sleptons, and the fermionic partner of the vector bosons which are a type of vector matter field. For instance, the supersymmetric version of quantum electrodynamics involves a vector supermultiplet whose contents are a massless photon and its spin-1/2 superpartner, the photino $[16$, 20].

Indeed theoretical formulation of supersymmetric gauge vector field has been largely studied [16, 22-24]. It was shown that gauge vector field component emerges from nonchiral scalar superfields when one uses some suitable constraint (Wess-Zumino) to remove exceeding nonphysical components fields $[20,22]$. Nevertheless, there is a lack of studies on models that describe supersymmetric vector matter fields. Therefore, one of the aims of this work is the attempt to address this lack in order to further study the interactions which can be involved. To this purpose we construct a formulation in which the vector field $B_{\mu}$ is complex and massive, but with no local charge, which we simply called "chargeless." Indeed as a matter field it transforms by the global $U(1)$ group [3]; that is, we would emphasize that $B_{\mu}$ is not a local gauge field but a free Proca-type one. Such models are interesting in order to contribute to the understanding of the supersymmetric model of electroweak theory though it contains charged vector particles. Furthermore it can improve our knowledge of the form of nuclear atomic structure and its interaction at high energy. Further, taking this model in a fundamental scenario of the strings theory [25], complex vector fields with matter symmetry are relevant to the vacuum polarization theory that can be connected to models which deal with Lorentz symmetry violation in high energy physics [26-46]. Another aim of this work is to obtain the supermultiplet that will accommodate a charged matter vector field and its supersymmetric partners and also to get the most appropriate supersymmetric action for this field which will be the subject of a forthcoming work. To this purpose we are going to formulate a supersymmetric Lagrangian starting from chargeless nonchiral superfield which contains the vector (matter) field. The present paper is outlined as follows: in Section 2, we present a model that acommodates two real vector matter fields; in Section 3 we compose the previous model in a complex form and we present the Dirac superspinor field $\Psi$; in Section 4 we present a general conclusion.

\section{Two Chargeless Vector Matter Superfields Model}

We are going to present a chargeless (real) formulation for vector matter field. To this aim we start from a general nonchiral scalar superfield which includes in the matter multiplet a vector field as irreducible representation of the Lorentz group. In order to build more ahead a complex extension we introduce two chargeless real scalar superfields doubling the number of degrees of freedom, which are written as

$$
\begin{aligned}
& \Phi\left(x^{\mu}, \theta_{a}, \bar{\theta}_{\dot{a}}\right)=C(x)+\theta^{a} \varphi_{a}(x)+\bar{\theta}^{\dot{a}} \bar{\varphi}_{\dot{a}}(x) \\
& +\theta^{2}[m(x)+i n(x)] \\
& +\bar{\theta}^{2}[m(x)-i n(x)] \\
& +\theta^{2} \bar{\theta}^{\dot{a}}\left[\bar{\lambda}_{\dot{a}}(x)-\frac{i}{2} \sigma_{a \dot{a}}^{\mu} \partial_{\mu} \varphi^{a}(x)\right] \\
& +\bar{\theta}^{2} \theta^{a}\left[\lambda_{a}(x)-\frac{i}{2} \sigma_{a \dot{a}}^{\mu} \partial_{\mu} \bar{\varphi}^{\dot{a}}\right] \\
& +\theta^{a} \sigma_{a \dot{a}}^{\mu} \bar{\theta}^{\dot{a}} X_{\mu}(x) \\
& +\theta^{2} \bar{\theta}^{2}\left[D(x)-\frac{1}{4} \square C(x)\right], \\
& \Lambda\left(x^{\mu}, \theta_{a}, \bar{\theta}_{\dot{a}}\right)=A(x)+\theta^{a} \chi_{a}(x)+\bar{\theta}^{\dot{a}} \bar{\chi}_{\dot{a}}(x) \\
& +\theta^{2}[\rho(x)+i \tau(x)] \\
& +\bar{\theta}^{2}[\rho(x)-i \tau(x)] \\
& +\theta^{2} \bar{\theta}^{\dot{a}}\left[\bar{\zeta}_{\dot{a}}(x)-\frac{i}{2} \sigma^{\mu}{ }_{a \dot{a}} \partial_{\mu} \chi^{a}(x)\right] \\
& +\bar{\theta}^{2} \theta^{a}\left[\zeta_{a}(x)-\frac{i}{2} \sigma_{a \dot{a}}^{\mu} \partial_{\mu} \bar{\chi}^{\dot{a}}(x)\right] \\
& +\theta^{a} \sigma_{a \dot{a}}^{\mu} \bar{\theta}^{\dot{a}} Y_{\mu}(x) \\
& +\theta^{2} \bar{\theta}^{2}\left[S(x)-\frac{1}{4} \square A(x)\right],
\end{aligned}
$$

where the superfields $\Phi$ and $\Lambda$ are particular constructions of matter vector supermultiplet which include real vector fields $X_{\mu}(x)$ and $Y_{\mu}(x)$ with helicity \pm 1 ; the fields $\varphi_{a}(x), \lambda_{a}(x)$, $\chi_{a}(x)$, and $\zeta_{a}(x)$ are two-component Weyl fermions with helicity $\pm 1 / 2$; and the fields $C(x), D(x), A(x), S(x), m(x)$, $n(x), \rho(x)$, and $\tau(x)$ are real scalar fields with spin-0. It is easy to verify that to both superfields the number of bosonic and fermionic degrees of freedom is the same. We stress that we only have applied the reality condition on the superfields which does not spoil the matter structure of these multiplets. Therefore the dynamics to chargeless supersymmetric vector fields can be obtained through suitable field-strengths which accommodate the real superfields $\Phi$ and $\Lambda$.

In order to construct the supersymmetric field-strengths for the real superfields $\Phi$ and $\Lambda$, which we call chargeless supersymmetric field-strengths, we are going to apply supersymmetric covariant derivatives on the above scalar superfields which result in chiral superfields, in such way that

$$
\begin{aligned}
& W_{a}=-\frac{1}{4} \bar{D} \bar{D} D_{a} \Phi\left(x^{\mu}, \theta_{a}, \bar{\theta}_{\dot{a}}\right), \\
& \bar{W}_{\dot{a}}=-\frac{1}{4} D D \bar{D}_{\dot{a}} \Phi\left(x^{\mu}, \theta_{a}, \bar{\theta}_{\dot{a}}\right),
\end{aligned}
$$


and, by similarity for $\Lambda$, we have that

$$
\begin{aligned}
& \Omega_{a}=-\frac{1}{4} \bar{D} \bar{D} D_{a} \Lambda\left(x^{\mu}, \theta_{a}, \bar{\theta}_{\dot{a}}\right), \\
& \bar{\Omega}_{\dot{a}}=-\frac{1}{4} D D \bar{D}_{\dot{a}} \Lambda\left(x^{\mu}, \theta_{a}, \bar{\theta}_{\dot{a}}\right),
\end{aligned}
$$

the $W_{a}$ and $\Omega_{a}$ are chiral spinor superfields.

We can redefine the superfields in the chiral superspace coordinates, namely, $\Phi\left(y^{\mu}, \theta_{a}\right), \Phi\left(z^{\mu}, \bar{\theta}_{\dot{a}}\right), \Lambda\left(y^{\mu}, \theta_{a}\right)$, and $\Lambda\left(z^{\mu}, \bar{\theta}_{\dot{a}}\right)$, such that $y^{\mu}=x^{\mu}+i \theta \sigma^{\mu} \bar{\theta}$ and $z^{\mu}=x^{\mu}-i \theta \sigma^{\mu} \bar{\theta}$. Hence the supersymmetric covariant derivatives are defined as

$$
\begin{aligned}
& D_{a}=\frac{\partial}{\partial \theta_{a}}+2 i \sigma^{\mu}{ }_{a \dot{a}} \bar{\theta}^{\dot{a}} \frac{\partial}{\partial y^{\mu}} \\
& \bar{D}_{\dot{a}}=-\frac{\partial}{\partial \bar{\theta}_{\dot{a}}}-2 i \theta^{a} \sigma^{\mu}{ }_{a \dot{a}} \frac{\partial}{\partial z^{\mu}} .
\end{aligned}
$$

According to these definitions we can compute the fieldstrengths $W_{a}$ and $\Omega_{a}$, and we have

$$
\begin{aligned}
W_{a}(y, \theta)= & \lambda_{a}(y)+2 \theta_{a} D(y)+\left(\sigma^{\mu \nu} \theta\right)_{a} X_{\mu \nu}(y) \\
& -i \theta^{2} \sigma^{\mu}{ }_{a \dot{b}} \partial_{\mu} \bar{\lambda}^{\dot{b}}(y), \\
\Omega_{a}(y, \theta)= & \zeta_{a}(y)+2 \theta_{a} S(y)+\left(\sigma^{\mu \nu} \theta\right)_{a} Y_{\mu \nu}(y) \\
& -i \theta^{2} \sigma^{\mu}{ }_{a \dot{b}} \partial_{\mu} \bar{\zeta}^{\dot{b}}(y),
\end{aligned}
$$

and in a similar way we can compute the field-strengths $\bar{W}_{\dot{a}}$ and $\bar{\Omega}_{\dot{a}}$. So we are in conditions to construct the supersymmetric model in terms of the superfields $W_{a}$ and $\Omega_{a}$ where chargeless vector matter field is present. The kinetic part can be written as

$$
\begin{gathered}
S_{\text {kin }}=\int d^{4} x\left\{d^{2} \theta\left(W_{a} W^{a}+\Omega_{a} \Omega^{a}\right)\right. \\
\left.+d^{2} \bar{\theta}\left(\bar{W}_{\dot{a}} \bar{W}^{\dot{a}}+\bar{\Omega}_{\dot{a}} \bar{\Omega}^{\dot{a}}\right)\right\} .
\end{gathered}
$$

We have adopted the usual conventions for the spinor algebra, for the superspace parametrization, and the translation invariance of the integral in the chiral coordinates [16, 22]. Then we obtain that expression (6) has the following component expansion:

$$
\begin{aligned}
& S_{\text {kin }}=\int d^{4} x\left\{-X_{\mu \nu}(x) X^{\mu \nu}(x)-Y_{\mu \nu}(x) Y^{\mu \nu}(x)\right. \\
& -4 i \lambda^{a}(x) \sigma^{\mu}{ }_{a \dot{b}} \partial_{\mu} \bar{\lambda}^{\dot{b}}(x)-4 i \zeta^{a}(x) \sigma^{\mu}{ }_{a \dot{b}} \partial_{\mu} \bar{\zeta}^{\dot{b}(x)} \\
& \left.+8 D^{2}(x)+8 S^{2}(x)\right\} .
\end{aligned}
$$

Action (7) describes the kinetic part of supersymmetric chargeless vector field. However, to write the full action that corresponds to the underlined field theory, we can also consider the supersymmetric mass term, given by

$$
\begin{aligned}
S_{m} & =\int d^{4} x d^{2} \theta \alpha^{2}\left[\Phi^{2}+\Lambda^{2}\right]=\int d^{4} x \alpha^{2}[C(x) D(x) \\
& -\frac{1}{4} C(x) \square C(x)+\varphi_{a}(x) \lambda^{a}(x)+\bar{\varphi}_{\dot{a}}(x) \bar{\lambda}^{\dot{a}}(x) \\
& -i \bar{\varphi}^{\dot{a}} \bar{\sigma}^{\mu}{ }_{a b} \partial_{\mu} \varphi^{b}+M(x)^{2}+N(x)^{2}+\frac{1}{4} X^{\mu} X_{\mu} \\
& +\frac{1}{4} Y^{\mu} Y_{\mu}+A(x) S(x)-\frac{1}{4} A(x) \square A(x) \\
& +\chi_{a}(x) \zeta^{a}(x)+\bar{\chi}_{\dot{a}}(x) \bar{\zeta}^{\dot{a}}(x)-i \bar{\chi}^{\dot{a}} \bar{\sigma}^{\mu}{ }_{\dot{a} b} \partial_{\mu} \chi^{b} \\
& \left.+\rho(x)^{2}+\tau(x)^{2}\right],
\end{aligned}
$$

where $\alpha^{2}$ is the mass parameter. As usual, the "mass" part of the action presents kinetic terms, beyond the usual mass terms, which were eliminated by spinor chirality property of superfields $W_{a}$ and $\Omega_{a}$. Furthermore, we could infer that the mass term in action (8) arises as a dynamical complement to the supersymmetric vector matter fields. Indeed supersymmetric matter-like fields are formulated with chiral spinor superfields.

\section{The Chargeless Complex Vector Matter Superfield Model}

We know that the supersymmetric action for two free vector matter fields might be built through nonchiral scalar superfields [22]. Moreover, we can see that the degrees of freedom of this model are compatible with the dynamical free fields in complex space $\mathbb{C}$. In this section our aim is to derive the appropriated complex superfield to describe supersymmetric complex vector fields. To this aim we need to strongly define two complex nonchiral scalar superfields, defined as

$$
\begin{aligned}
\Sigma\left(x_{\mu}, \theta_{a}, \bar{\theta}_{\dot{a}}\right)= & k(x)+\theta^{a} \xi_{a}(x)+\bar{\theta}^{\dot{a}} \bar{C}_{\dot{a}}(x)+\theta^{2} l(x) \\
& +\bar{\theta}^{2} f(x) \\
& +\theta^{2} \bar{\theta}^{\dot{a}}\left[\bar{G}_{\dot{a}}(x)-\frac{i}{2} \sigma^{\mu}{ }_{a \dot{a}} \partial_{\mu} \xi^{a}(x)\right] \\
& +\bar{\theta}^{2} \theta^{a}\left[R_{a}(x)-\frac{i}{2} \sigma^{\mu}{ }_{a \dot{a}} \partial_{\mu} \bar{C}^{\dot{a}}\right] \\
& +\theta^{a} \sigma^{\mu}{ }_{a \dot{a}} \bar{\theta}^{\dot{a}} B_{\mu}(x) \\
& +\theta^{2} \bar{\theta}^{2}\left[d(x)-\frac{1}{4} \square k(x)\right] . \\
K\left(x_{\mu}, \theta_{a}, \bar{\theta}_{\dot{a}}\right)= & a(x)+\theta^{a} T_{a}(x)+\bar{\theta}^{\dot{a}} \bar{H}_{\dot{a}}(x) \\
& +\theta^{2} j(x)+\bar{\theta}^{2} E(x) \\
& +\theta^{2} \bar{\theta}^{\dot{a}}\left[\bar{Q}_{\dot{a}}(x)-\frac{i}{2} \sigma^{\mu}{ }_{a \dot{a}} \partial_{\mu} T^{a}(x)\right]
\end{aligned}
$$




$$
\begin{aligned}
& +\bar{\theta}^{2} \theta^{a}\left[U_{a}(x)-\frac{i}{2} \sigma_{a \dot{a}}^{\mu} \partial_{\mu} \bar{H}^{\dot{a}}(x)\right] \\
& +\theta^{a} \sigma_{a \dot{a}}^{\mu} \bar{\theta}^{\dot{a}} Z_{\mu}(x) \\
& +\theta^{2} \bar{\theta}^{2}\left[v(x)-\frac{1}{4} \square a(x)\right],
\end{aligned}
$$

and in a similar way we can define the complex conjugated superfields.

Superfields (9) and (10) present multiplets with complex vector fields $B_{\mu}(x)$ and $Z_{\mu}(x)$ and spin-1; the $\xi_{a}(x), \bar{C}_{\dot{a}}(x)$, $R_{a}(x), \bar{G}_{\dot{a}}(x), T_{a}(x), \bar{H}_{\dot{a}}(x), U_{a}(x)$, and $\bar{Q}_{\dot{a}}(x)$ are Weyl fermion fields with spin-1/2; and the $k(x), d(x), a(x)$, and $v(x)$ are complex scalar fields with spin-0. So the rule that implies an invariant mechanism is

$$
K=i \Sigma^{\dagger}
$$

We observe that transformation rule (11) guarantees writing a consistent kinetic term for the complex vector field without breaking the global $U(1)$ gauge symmetry. Another advantage that came to light is that transformations (11) eliminate the exceeding fields which does not contribute for the supersymmetric action, which allows bosons and fermions to have the same physical degrees of freedom. Indeed the constraint relation to the superfields implies the relations of the component fields as follows:

$$
\begin{aligned}
a(x) & =i k^{*}(x), \\
T_{a}(x) & =i C_{a}(x), \\
v(x) & =i d^{*}(x), \\
\bar{H}_{a}(x) & =i \bar{\xi}_{a}(x), \\
j(x) & =i f^{*}(x), \\
U_{a}(x) & =i G_{a}(x), \\
E(x) & =i l^{*}(x), \\
\bar{Q}_{a}(x) & =i \bar{R}_{a}(x), \\
Z_{\mu}(x) & =i B_{\mu}^{*}(x) .
\end{aligned}
$$

So, we can adjust the complex extension of the chargeless superfields $\Phi$ and $\Lambda$ by assuming the equation $\Sigma=\Phi+i \Lambda$, where we find the following relation of fields:

$$
\begin{aligned}
k(x) & =C(x)+i A(x), \\
\xi_{a}(x) & =\varphi_{a}(x)+i \chi_{a}(x), \\
\bar{C}_{a}(x) & =\bar{\varphi}_{a}(x)+i \bar{\chi}_{a}(x), \\
l(x) & =m(x)+n(x)+i(\rho(x)+\tau(x)), \\
\bar{G}_{a}(x) & =\bar{\lambda}_{a}(x)+i \bar{\zeta}_{a}(x),
\end{aligned}
$$

$$
\begin{aligned}
f(x) & =m(x)+n(x)-i(\rho(x)+\tau(x)), \\
R_{a}(x) & =\lambda_{a}(x)+i \zeta_{a}(x), \\
B_{\mu}(x) & =X_{\mu}(x)+i Y_{\mu}(x), \\
d(x) & =D(x)+i S(x) .
\end{aligned}
$$

To describe the dynamics of the supersymmetric complex vector fields with matter symmetry we need to construct an appropriated complex supersymmetric field-strength model in order to accommodate superfields $\Sigma$ and $K$. This can be reached starting from the following definitions:

$$
\begin{aligned}
& \Upsilon_{a}=-\frac{1}{4} \bar{D} \bar{D} D_{a} \Sigma\left(x_{\mu}, \theta_{a}, \bar{\theta}_{\dot{a}}\right), \\
& \bar{\Upsilon}_{\dot{a}}=-\frac{1}{4} D D \bar{D}_{\dot{a}} \Sigma^{\dagger}\left(x_{\mu}, \theta_{a}, \bar{\theta}_{\dot{a}}\right), \\
& \Gamma_{a}=-\frac{1}{4} \bar{D} \bar{D} D_{a} K\left(x_{\mu}, \theta_{a}, \bar{\theta}_{\dot{a}}\right), \\
& \bar{\Gamma}_{\dot{a}}=-\frac{1}{4} D D \bar{D}_{\dot{a}} K^{\dagger}\left(x_{\mu}, \theta_{a}, \bar{\theta}_{\dot{a}}\right),
\end{aligned}
$$

where $\Upsilon_{a}$ and $\Gamma_{a}$ are charged spinor superfields. As a consequence of the complex extension procedure we must relate chargeless spinor superfields $\Omega_{a}$ and $W_{a}$ with the complex definitions (14) which, in the simplest way, is

$$
\begin{aligned}
& \Upsilon_{a}=W_{a}+i \Omega_{a}, \\
& \bar{\Upsilon}_{\dot{a}}=\bar{W}_{\dot{a}}-i \bar{\Omega}_{\dot{a}}, \\
& \Gamma_{a}=\Omega_{a}+i W_{a}, \\
& \bar{\Gamma}_{\dot{a}}=\bar{\Omega}_{\dot{a}}-i \bar{W}_{\dot{a}},
\end{aligned}
$$

and by assuming the spinor identities $W_{a} \Omega^{a}=\Omega_{a} W^{a}$ and $\bar{W}_{\dot{a}} \bar{\Omega}^{\dot{a}}=\bar{\Omega}_{\dot{a}} \bar{W}^{\dot{a}}$ we can find the kinetic supersymmetric Lagrangian for the complex vector fields:

$$
\begin{aligned}
\mathscr{L}_{k} & =i\left(\bar{\Upsilon}_{\dot{a}} \bar{\Gamma}^{\dot{a}}-\Upsilon_{a} \Gamma^{a}\right) \\
& =W_{a} W^{a}+\bar{W}_{\dot{a}} \bar{W}^{\dot{a}}+\Omega_{a} \Omega^{a}+\bar{\Omega}_{\dot{a}} \bar{\Omega}^{\dot{a}} .
\end{aligned}
$$

We can observe that the left-hand side of the latter equation is the complex extension of chargeless Lagrangian (7) that was written in terms of charged spinor superfields. Bearing this in mind, we can then redefine the kinetic Lagrangian (16) simply by combining the charged spinor superfields $\Upsilon_{a}$ and $\Gamma_{a}$ as a "Dirac superspinor" $\Psi$, such that

$$
\Psi\left(x^{\mu}, \theta_{a}, \bar{\theta}_{\dot{a}}\right)=\left(\begin{array}{c}
\Upsilon_{a} \\
\bar{\Gamma}^{\dot{a}}
\end{array}\right),
$$

and also we assume $\bar{\Psi}$ to be the adjoint Dirac superspinor representation. 
In this case we have that $\bar{\Psi}=\Psi^{\dagger} \gamma^{0}=\left(\Gamma^{a} \bar{\Upsilon}_{\dot{a}}\right)$, and so the supersymmetric action from the kinetic Lagrangian (16) is now given by

$$
\begin{aligned}
S_{k} & =\int d^{4} x d^{2} \theta d^{2} \bar{\theta}(i \bar{\Psi} \Psi) \\
& =i \int d^{4} x d^{2} \theta d^{2} \bar{\theta}\left(\bar{\Upsilon}_{\dot{a}} \bar{\Gamma}^{\dot{a}}-\Upsilon_{a} \Gamma^{a}\right) .
\end{aligned}
$$

We can note that the product of Dirac superspinors $\bar{\Psi} \Psi$ obeys matter symmetry and it presents an interesting analogy to charged scalar superfield product $S^{\dagger} S$. In this sense we verify that $\Psi$ and $\bar{\Psi}$ represent two chiral supersymmetric extensions for the matter vector field which can be transformed under $U(1)$ global gauge group in the following way:

$$
\begin{aligned}
& \Psi^{\prime}=e^{-2 i q \beta} \Psi, \\
& \bar{\Psi}^{\prime}=\bar{\Psi} e^{2 i q \beta^{\dagger}},
\end{aligned}
$$

where $\beta$ is a global $U(1)$ gauge parameter and $q$ is the charge of the global symmetry. We can emphasize that the expressions (19) represent that each of the components of multiplet (17) has the same symmetry. So action (18) is then invariant under transformations (19). In order to obtain the component Lagrangian we can expand the product $\bar{\Psi}$ by considering that

$$
\begin{aligned}
\Upsilon_{a}(y, \theta)= & R_{a}(y)+2 \theta_{a} d(y)+\left(\sigma^{\mu \nu} \theta\right)_{a} F_{\mu \nu}(y) \\
& -i \theta^{2} \sigma^{\mu}{ }_{a b} \partial_{\mu} \bar{G}^{\dot{b}}(y), \\
\Gamma_{a}(y, \theta)= & U_{a}(y)+2 \theta_{a} v(y)+\left(\sigma^{\mu \nu} \theta\right)_{a} Z_{\mu \nu}(y) \\
& -i \theta^{2} \sigma^{\mu}{ }_{a b} \partial_{\mu} \bar{Q}^{\dot{b}}(y),
\end{aligned}
$$

and similarly for $\bar{Y}_{\dot{a}}$ and $\bar{\Gamma}_{\dot{a}}$.

We note the presence of the complex matter fieldstrengths; namely,

$$
\begin{aligned}
& F_{\mu \nu}=\partial_{\mu} B_{\nu}-\partial_{\nu} B_{\mu} ; \\
& Z_{\mu \nu}=\partial_{\mu} Z_{\nu}-\partial_{\nu} Z_{\mu}
\end{aligned}
$$

hence action (18) can be expanded and we obtain

$$
\begin{aligned}
S_{k} & =\int d^{4} x\left\{\frac{i}{2} F_{\mu \nu}(x) Z^{\mu \nu}(x)-\frac{i}{2} F_{\mu \nu}^{*}(x) Z^{* \mu \nu}(x)\right. \\
& -R^{a}(x) \sigma^{\mu}{ }_{a b} \partial_{\mu} \bar{Q}^{\dot{b}}(x)-U^{a}(x) \sigma_{a \dot{b}}^{\mu} \partial_{\mu} \bar{G}^{\dot{b}}(x) \\
& +\bar{R}^{\dot{a}}(x) \bar{\sigma}^{\mu}{ }_{a b} \partial_{\mu} Q^{b}(x)+\bar{U}^{a}(x) \bar{\sigma}^{\mu}{ }_{a b} \partial_{\mu} G^{b}(x) \\
& \left.+4 i v(x) d(x)-4 i v^{*}(x) d^{*}(x)\right\} .
\end{aligned}
$$

In this format we can recognize the dynamical term that describes the matter vector field as $(i / 2) F_{\mu \nu} Z^{\mu \nu}-$ $(i / 2) F_{\mu \nu}^{*} Z^{* \mu \nu}$. It involves both $F_{\mu \nu}$ and $Z_{\mu \nu}$ matter tensors.
However, it does not correspond to the conventional kinetic term for the matter vector field, and action (18) shows more degrees of freedom than necessary. In order to get rid of such fields we must assume the rule of transformation (11) which is a constraint of half of the degrees and consequently action (18) reaches the correct number of component fields.

Applying condition (11) in action (22) we can reach the usual dynamical matter field strength term, or

$$
\frac{i}{2} F_{\mu \nu} Z^{\mu \nu}-\frac{i}{2} F^{*}{ }_{\mu \nu} Z^{* \mu \nu} \Longrightarrow-F^{*}{ }_{\mu \nu} F^{\mu \nu},
$$

and so the $Z^{\mu \nu}$ tensor field is reabsorbed in this action. Likewise and without loss of generality, we could have chosen the inverse relation $\Sigma=i K^{\dagger}$ what implies reabsorbing the $F^{\mu \nu}$ tensor field. Then by using the whole relation (12) in action (18) we find that the complex supersymmetric model for the matter vector field can be written as

$$
\begin{aligned}
S_{k} & =\int d^{4} x\left\{-F^{*}{ }_{\mu \nu}(x) F^{\mu \nu}(x)\right. \\
& -2 i R^{a}(x) \sigma^{\mu}{ }_{a \dot{b}} \partial_{\mu} \bar{R}^{\dot{b}}(x)-2 i G^{a}(x) \sigma^{\mu}{ }_{a \dot{b}} \partial_{\mu} \bar{G}^{\dot{b}}(x) \\
& \left.+8 d^{*}(x) d(x)\right\},
\end{aligned}
$$

where expression $-F^{*}{ }_{\mu \nu}(x) F^{\mu \nu}(x)$ represents the usual kinetic term of the vector matter field while the terms represent with the components $R^{a}$ and $G^{a}$ the fermionic sector, and the last term corresponds to the auxiliary field $d$ term. To completeness we are going to introduce the massive action term in the model. Observing the symmetries of nonchiral fields $\Sigma$ and $K$ the massive supersymmetric term can be suitable defined as

$$
S_{m}=\frac{\alpha^{2}}{2} \int d^{4} x d^{2} \theta d^{2} \bar{\theta}\left[\Sigma^{\dagger} \Sigma+K^{\dagger} K\right],
$$

where $\alpha^{2}$ is a mass parameter. From nonchiral superfields $\Sigma$ and $K$ we can obtain the massive vector matter field term $B^{*}{ }_{\mu} B^{\mu}$ as well as their supersymmetric partners. In order to perform it we are going to compute action (25) by employing condition $K=i \Sigma^{\dagger}$ where one has that $(1 / 2) \Sigma^{\dagger} \Sigma+(1 / 2) K^{\dagger} K=$ $\Sigma^{\dagger} \Sigma$, and by applying definition (10) the full supersymmetric matter vector field model can be then obtained from Dirac superspinor field $\Psi$ associated to the nonchiral scalar fields $\Sigma$ and $K$ in the following form:

$$
S=S_{k}+S_{m}=\int d^{4} x d^{2} \theta d^{2} \bar{\theta}\left(i \bar{\Psi} \Psi+\alpha^{2} \Sigma^{\dagger} \Sigma\right),
$$

where the mass part of action can be obtained in component fields as

$$
\begin{aligned}
S_{m} & =\alpha^{2} \int d^{4} x\left(d^{*}(x) k(x)+d(x) k^{*}(x)\right. \\
& -\frac{1}{4} k^{*}(x) \square k(x)-\frac{1}{4} k(x) \square k^{*}(x)+\xi_{a}(x) G^{a}(x) \\
& +\bar{\xi}_{\dot{a}}(x) \bar{G}^{\dot{a}}(x)+C_{a}(x) R^{a}(x)+\bar{C}_{\dot{a}}(x) \bar{R}^{\dot{a}}(x)
\end{aligned}
$$




$$
\begin{aligned}
& -2 i \bar{\xi}^{\dot{a}}(x) \bar{\sigma}_{a b}^{\mu} \partial_{\mu} \xi^{b}(x)-2 i \bar{C}^{\dot{a}}(x) \bar{\sigma}_{a b}^{\mu} \partial_{\mu} C^{b}(x) \\
& \left.+B_{\mu}^{*} B^{\mu}+f^{*}(x) f(x)+l^{*}(x) l(x)\right) .
\end{aligned}
$$

Here we observe the mass term to $B^{\mu}, f(x)$, and $l(x)$ fields. As in the usual supersymmetric models we note that mass action (27) also contributes to kinetic structure, namely, with the terms $-(1 / 4) k^{*}(x) \square k(x),-2 i \bar{\xi}^{\dot{a}} \bar{\sigma}^{\mu}{ }_{a b} \partial_{\mu} \xi^{b}$, and $-2 i \bar{C}^{\dot{a}} \bar{\sigma}^{\mu}{ }_{a b} \partial_{\mu} C^{b}$. The action also shows mixing mass scalar and fermionic terms, namely, $d^{*}(x) k(x), d(x) k^{*}(x)$, $\xi_{a}(x) G^{a}(x), \bar{\xi}_{\dot{a}}(x) \bar{G}^{\dot{a}}(x), C_{a}(x) R^{a}(x)$, and $\bar{C}_{\dot{a}}(x) \bar{R}^{\dot{a}}(x)$. By verifying the presence of extra kinetic terms in (27) what can suggest that when we particularly treat supersymmetric matter vector fields the mass action contributes with a "dynamic complement" to the kinetic action (24). Furthermore, we remark that mass action (27) is important to match the number of bosonic and fermionic degrees of freedom of the supersymmetric matter vector action (26) for the consistency of the model. We can redefine the component fields absorbing the mass parameter as follows:

$$
\begin{gathered}
k(x) \longrightarrow \frac{1}{\alpha} k(x), \\
\xi_{a}(x) \longrightarrow \frac{1}{\alpha} \xi_{a}(x), \\
C_{a}(x) \longrightarrow \frac{1}{\alpha} C_{a}(x), \\
f(x) \longrightarrow \frac{1}{\alpha} f(x), \\
l(x) \longrightarrow \frac{1}{\alpha} l(x) .
\end{gathered}
$$

So the action can be rewritten as

$$
\begin{aligned}
S_{\mathrm{ek}} & =\frac{1}{2} \int d^{4} x\left(-F_{\mu \nu}^{*}(x) F^{\mu \nu}(x)\right. \\
& -2 i R^{a}(x) \sigma^{\mu}{ }_{a \dot{b}} \partial_{\mu} \bar{R}^{\dot{b}}(x)-2 i G^{a}(x) \sigma_{a \dot{b}}^{\mu} \partial_{\mu} \bar{G}^{\dot{b}}(x) \\
& -\frac{1}{4} k^{*}(x) \square k(x)-\frac{1}{4} k(x) \square k^{*}(x) \\
& +-2 i \bar{\xi}^{\dot{a}}(x) \bar{\sigma}^{\mu}{ }_{a b} \partial_{\mu} \xi^{b}(x)-2 i \bar{C}^{\dot{a}}(x) \bar{\sigma}^{\mu}{ }_{a b} \partial_{\mu} C^{b}(x) \\
& \left.+8 d^{*}(x) d(x)+f^{*}(x) f(x)+l^{*}(x) l(x)\right) ;
\end{aligned}
$$

analogously the mass action is now given by

$$
\begin{aligned}
S_{\mathrm{em}} & =\int d^{4} x\left\{\alpha^{2} B^{*}{ }_{\mu} B^{\mu}+\alpha d^{*}(x) k(x)\right. \\
& +\alpha d(x) k^{*}(x)+\alpha \xi_{a}(x) G^{a}(x)+\alpha \bar{\xi}_{\dot{a}}(x) \bar{G}^{\dot{a}}(x) \\
& \left.+\alpha C_{a}(x) R^{a}(x)+\alpha \bar{C}_{\dot{a}}(x) \bar{R}^{\dot{a}}(x)\right\},
\end{aligned}
$$

so the complex scalar fields $d(x), f(x)$, and $l(x)$ have no dynamics and arise as auxiliary fields. Thus assuming the action (26) to be the sum of the redefined actions (29) and (30) and rearranging (we adopt the Weyl representation to the gamma matrices) the (Dirac) spinor fields $\Theta$ and $\Pi$, The sum of the action (29) and the action (30) results in an offshell action $S_{\text {os }}$ written in the following form

$$
\begin{aligned}
S_{\mathrm{os}} & =\int d^{4} x\left\{-\frac{1}{2} F_{\mu \nu}^{*}(x) F^{\mu \nu}(x)+\frac{1}{4} \partial_{\mu} k^{*}(x) \partial^{\mu} k(x)\right. \\
& -i \bar{\Theta}(x) \gamma^{\mu} \partial_{\mu} \Theta(x)-i \bar{\Pi}(x) \gamma^{\mu} \partial_{\mu} \Pi(x) \\
& +\alpha \bar{\Theta}(x) \gamma^{5} \Theta(x)+\alpha \bar{\Pi}(x) \gamma^{5} \Pi(x)+4 d^{*}(x) d(x) \\
& +\frac{1}{2} f^{*}(x) f(x)+\frac{1}{2} l^{*}(x) l(x)+\alpha^{2} B^{*}{ }_{\mu} B^{\mu} \\
& \left.+\alpha d^{*}(x) k(x)+\alpha d(x) k^{*}(x)\right\},
\end{aligned}
$$

where we denote the Dirac spinors of mass $\alpha$ as

$$
\begin{aligned}
& \Theta(x)=\left(\begin{array}{c}
\xi_{a} \\
\bar{G}^{\dot{a}}
\end{array}\right), \\
& \Pi(x)=\left(\begin{array}{c}
C_{a} \\
\bar{R}^{\dot{a}}
\end{array}\right) .
\end{aligned}
$$

For action (31) we have obtained chiral spinor mass terms given by $\alpha \bar{\Theta}(x) \gamma^{5} \Theta(x)$ and $\alpha \bar{\Pi}(x) \gamma^{5} \Pi(x)$. From the action (31) we can obtain the equation of motion for the fields:

$$
\begin{array}{r}
\partial_{\mu} F^{\mu \nu}(x)+\alpha^{2} B^{\nu}(x)=0, \\
\gamma^{\mu} \partial_{\mu} \Theta(x)-\alpha \gamma^{5} \Theta(x)=0, \\
\gamma^{\mu} \partial_{\mu} \Pi(x)-\alpha \gamma^{5} \Pi(x)=0, \\
\square k(x)+\alpha^{2} k(x)=0,
\end{array}
$$

with $f(x)=l(x)=0$. Taking off-shell action (31) we note that it has 16 bosonic degrees of freedom concerning the matter fields $B_{\mu}(x), k(x), d(x), f(x)$, and $l(x)$ and their complex conjugated ones, as well as 16 fermionic degrees of freedom for the Dirac spinor fields $\Theta(x)$ and $\Pi(x)$ and their conjugated complex ones, which is consistent with the supersymmetry. From equations of motion (33) we note that there are three auxiliary complex scalar fields $d(x), f(x)$, and $l(x)$ and massive dynamical complex scalar field $k(x)$. Moreover, as expected we have obtained a matter Proca-type equation for field $B_{\mu}$. In this context, it is interesting to note that from the on-shell action we can easily extract from action (31) the supersymmetric generalization of matter vector field; it is only possible if we include two dynamical Dirac chiral spinor fields $\Theta(x)$ and $\Pi(x)$ along a massive scalar field $k(x)$. Furthermore, a peculiar aspect of the spinor fields $\Theta(x)$ and $\Pi(x)$, in the present case, is that their mass terms (33) arise with chiral structure due to the presence of the matrix $\gamma^{5}$. 


\section{Conclusion}

In this paper, which is part of a program started in a previous work [32], we propose to formulate and analyze the simple dynamics of chargeless vector matter field in a supersymmetric scenario. There are three motivations for this proposal: the lack of this approach in the literature; getting a clue on the possibility of Lorentz symmetry violation in supersymmetric theories; and the role played by the simplest case of highspin field in field and string theories. To these aims we have started from real nonchiral scalar superfield in order to obtain real matter Proca-type field in a supersymmetric Lagrangian generalization [32]. In a straightforward way, the complex model was obtained extending the real scalar nonchiral superfields to the complex space [47-51].

The very interesting point in this enterprise is that, in order to obtain a complex Proca-type term, we face an ambiguous choice of which is the real or imaginary part of the field. This ambiguity reveals a symmetry which we represent as a kind of simple Hodge-duality symmetry, $K=i \Sigma^{\dagger}$. In fact, despite its apparent simplicity, it is essential to match the number of degrees of freedom of the fermionic and bosonic sectors and to compose the complex (chargeless) Procatype dynamical term with global $U(1)$ symmetry. As this symmetry appears by construction, it represents a different approach compared to Ferrara et al.s work [24]. On the other hand, as usual, the supersymmetric mass term is also important to compose the dynamics of the supersymmetric model and of its component fields.

We can conclude that, in addition to the supersymmetric mass term, to build supersymmetric matter chargeless vector field Lagrangian the Hodge-type duality symmetry is an essential ingredient to be further explored. We can also observe that, as the usual super-QED model, the complex dynamical Proca-type term emerges from a product of Dirac superspinors $\Psi \bar{\Psi}$. We can remark that the Dirac superspinor field $\Psi$ is also chiral because it is a combination of chiral Weyl superspinors $\Gamma^{a}$ and $\Upsilon^{a}$. Finally, we emphasize that on-shell supersymmetric action obtained (31) revealing two fermionic Dirac fields $\Theta(x)$ and $\Pi(x)$ and a massive scalar field $k(x)$ as supersymmetric partners associated to the complex vector field $B_{\mu}(x)$, which is the initial step to implement local gauge symmetry, charge, and interaction; we could anticipate that nonminimal couplings have an essential role, in a forthcoming work.

\section{Conflict of Interests}

The authors declare that they have no conflict of interests related to this paper.

\section{References}

[1] C. Itzykson and J.-B. Zuber, Quantum Field Theory, Dover Publications, 2005.

[2] P. Ramond, Field Theory: A Modern Primer, Westview Press, 2nd edition, 1990.

[3] N. N. Bogoliubov and D. V. Shirkov, Introduction to the Theory of Quantized Fields, John Wiley \& Sons, 3rd edition, 1980.
[4] P. Fayet and S. Ferrara, "Supersymmetry," Physics Reports, vol. 32, no. 5, pp. 249-334, 1977.

[5] H. P. Nilles, "Supersymmetry, supergravity and particle physics," Physics Reports, vol. 110, no. 1-2, pp. 1-162, 1984.

[6] P. D. D’Eath, “Canonical quantization of supergravity," Physical Review D, vol. 29, no. 10, pp. 2199-2219, 1984.

[7] J.-P. Ader, F. Biet, and Y. Noirot, "Supersymmetric structure of the induced W gravities," Classical and Quantum Gravity, vol. 16, no. 3, pp. 1027-1037, 1999.

[8] C. Kiefer, T. Luck, and P. Moniz, "Semiclassical approximation to supersymmetric quantum gravity," Physical Review. D, vol. 72, no. 4, Article ID 045006, 2005.

[9] A. Riotto, "More relaxed supersymmetric electroweak baryogenesis," Physical Review D, vol. 58, Article ID 095009, 1998.

[10] A. Riotto, "Supersymmetric electroweak baryogenesis, nonequilibrium field theory and quantum Boltzmann equations," Nuclear Physics B, vol. 518, no. 1-2, pp. 339-360, 1998.

[11] I. R. Klebanov and A. A. Tseytlin, "Gravity duals of supersymmetric $\mathrm{SU}(\mathrm{N}) \times \mathrm{SU}(\mathrm{N}+\mathrm{M})$ gauge theories," Nuclear Physics $B$, vol. 578, no. 1-2, pp. 123-138, 2000.

[12] P. H. Chankowski, A. Falkowski, S. Pokorski, and J. Wagner, "Electroweak symmetry breaking in supersymmetric models with heavy scalar superpartners," Physics Letters, Section B: Nuclear, Elementary Particle and High-Energy Physics, vol. 598, no. 3-4, pp. 252-262, 2004.

[13] J. D. Edelstein and C. Núñez, "Supersymmetric electroweak cosmic strings," Physical Review D, vol. 55, no. 6, pp. 3811-3819, 1997.

[14] L. Girardello, A. Giveon, M. Porrati, and A. Zaffaroni, "NonAbelian strong-weak coupling duality in (string-derived) $N=4$ supersymmetric Yang-Mills theories," Physics Letters B, vol. 334, no. 3-4, pp. 331-338, 1994.

[15] J. Wess and J. Bagger, Supersymmetry and Supergravity, Princeton University Press, Princeton, NJ, USA, 2nd edition, 1992.

[16] D. Bailin and A. Love, Supersymmetric Gauge Field Theory and String Theory, IOP Publishing, 1994.

[17] S. Coleman and J. Mandula, "All possible symmetries of the S matrix," Physical Review, vol. 159, no. 5, pp. 1251-1256, 1967.

[18] S. J. Gates, M. T. Grisaru, M. Rocek, and W. Siegel, Superspace, Benjamin/Cummings, Reading, 1983.

[19] R. Haag, J. T. Lopuszanski, and M. Sohnius, "All possible generators of supersymmetries of the S-matrix," Nuclear Physics $B$, vol. 88, pp. 257-274, 1975.

[20] S. Weinberg, The Quantum Theory of Fields, vol. 3, Cambridge University Press, Cambridge, UK, 2000.

[21] J. Wess and B. Zumino, "Supergauge transformations in four dimensions," Nuclear Physics B, vol. 70, no. 1, pp. 39-50, 1974.

[22] H. J. W. Müller-Kirsten and A. Wiedemann, Supersymmetry, World Scientific, Singapore, 1987.

[23] S. J. Gates Jr., "Why auxiliary fields matter: the strange case of the $4-\mathrm{D}, N=1$ supersymmetric QCD effective action. 2," Nuclear Physics B, vol. 485, no. 1-2, pp. 145-184, 1997.

[24] S. Ferrara, L. Girardello, and F. Palumbo, "General mass formula in broken supersymmetry," Physical Review D, vol. 20, no. 2, pp. 403-408, 1979.

[25] V. A. Kostelecký and S. Samuel, "Spontaneous breaking of Lorentz symmetry in string theory," Physical Review D, vol. 39, no. 2, pp. 683-685, 1989.

[26] V. E. R. Lemes, A. L. M. A. Nogueira, and J. A. Helayël-Neto, "Supersymmetric generalization of the tensor matter fields," 
International Journal of Modern Physics A, vol. 13, no. 18, pp. 3145-3156, 1998.

[27] D. Colladay and V. A. Kostelecky, "CPT violation and the standard model," Physical Review D, vol. 55, pp. 6760-6774, 1997.

[28] D. Colladay and V. A. Kostelecký, "Lorentz-violating extension of the standard model," Physical Review D, vol. 58, no. 11, Article ID 116002, 1998.

[29] A. A. Andrianov and R. Soldati, "Lorentz symmetry breaking in Abelian vector-field models with Wess-Zumino interaction," Physical Review D, vol. 51, no. 10, pp. 5961-5964, 1995.

[30] A. A. Andrianov and R. Soldati, "Patterns of Lorentz symmetry breaking in QED by CPT-odd interaction," Physics Letters B, vol. 435, no. 3-4, pp. 449-452, 1998.

[31] S. M. Carrol, G. B. Field, and R. Jackiw, "Limits on a Lorentzand parity-violating modification of electrodynamics," Physical Review D, vol. 41, no. 4, pp. 1231-1240, 1990.

[32] L. P. Colatto, A. L. A. Penna, and W. C. Santos, "Charged tensor matter fields and Lorentz symmetry violation via spontaneous symmetry breaking," European Physical Journal C, vol. 36, no. 1, pp. 79-87, 2004.

[33] H. Belich, J. L. Boldo, L. P. Colatto, J. A. Helayel-Neto, and A. L. M. A. Nogueira, "Supersymmetric extension of the Lorentz- and CPT-violating Maxwell-Chern-Simons model," Physical Review D, vol. 68, Article ID 065030, 2003.

[34] H. Belich Jr., T. Costa-Soares, M. M. Ferreira Jr., J. A. HelayëlNeto, and M. T. D. Orlando, "Lorentz-symmetry violation and electrically charged vortices in the planar regime," International Journal of Modern Physics A, vol. 21, no. 11, pp. 2415-2429, 2006.

[35] M. M. Ferreira Jr., "Electron-electron interaction in a MaxwellChern-Simons model with a purely spacelike Lorentz-violating background," Physical Review D, vol. 71, no. 4, Article ID 045003, 9 pages, 2005.

[36] H. Belich, T. Costa-Soares, M. M. Ferreira Jr., and J. A. HelayelNeto, "Non-minimal coupling to a Lorentz-violating background and topological implications," The European Physical Journal C, vol. 41, no. 3, pp. 421-426, 2005.

[37] M. B. Cantcheff, "Lorentz symmetry breaking and planar effects from non-linear electrodynamics," The European Physical Journal C-Particles and Fields, vol. 46, no. 1, pp. 247-254, 2006.

[38] H. Belich, M. A. De Andrade, and M. A. Santos, "Gauge theories with Lorentz-symmetry violation by symplectic projector method," Modern Physics Letters A, vol. 20, pp. 2305-2316, 2005.

[39] T. G. Rizzo, "Lorentz violation in extra dimensions," Journal of High Energy Physics, vol. 2005, no. 9, article 036, 2005.

[40] M. M. Ferreira Jr. and M. S. Tavares, "Nonrelativistic electronelectron interaction in a maxwell-chern-simons-proca model endowed with a timelike lorentz-violating background," International Journal of Modern Physics A, vol. 22, p. 1685, 2007.

[41] H. Belich, T. Costa-Soares, M. M. Ferreira Jr., J. A. HelayelNeto, and F. M. O. Mouchereck, "Lorentz-violating corrections on the hydrogen spectrum induced by a nonminimal coupling," Physical Review D, vol. 74, no. 6, Article ID 065009, 6 pages, 2006.

[42] J. Alfaro, A. A. Andrianov, M. Cambiaso, P. Giacconi, and R. Soldati, "On the consistency of Lorentz invariance violation in QED induced by fermions in constant axial-vector background," Physics Letters B, vol. 639, no. 5, pp. 586-590, 2006.

[43] L. P. Colatto, A. L. A. Penna, and W. C. Santos, "Vector solitons and spontaneous Lorentz violation mechanism," http://arxiv .org/abs/hep-th/0610096.
[44] M. M. Ferreira Jr. and A. R. Gomes, "Lorentz violating effects on a quantized two-level system," http://arxiv.org/abs/0803.4010.

[45] J. Alfaro, A. A. Andrianov, M. Cambiaso, P. Giacconi, and R. Soldati, "Bare and induced lorentz and cpt invariance violations in qed," International Journal of Modern Physics A, vol. 25, no. 16, pp. 3271-3306, 2010.

[46] T. G. Rizzo, "Lorentz violation in warped extra dimensions," Journal of High Energy Physics, vol. 2010, no. 11, article 156, 2010.

[47] J. Helayel-Neto, R. Jengo, F. Legovini, and S. Pugnetti, "Supersymmetry, Complex Structures And Superfields," SISSA38/87/EP.

[48] Y. Kobayashi and S. Nagamachi, "Generalized complex superspace-involutions of superfields," Journal of Mathematical Physics, vol. 28, no. 8, pp. 1700-1708, 1987.

[49] P. A. Grassi, G. Policastro, and M. Porrati, "Notes on the quantization of the complex linear superfield," Nuclear Physics $B$, vol. 597, no. 1-3, pp. 615-632, 2001.

[50] S. M. Kuzenko and S. J. Tyler, "Complex linear superfield as a model for Goldstino," Journal of High Energy Physics, vol. 2011, no. 4, article 57, 2011.

[51] S. J. Gates, J. Hallett, T. Hubsch, and K. Stiffler, "The real anatomy of complex linear superfields," International Journal of Modern Physics A, vol. 27, no. 24, Article ID 1250143, 2012. 

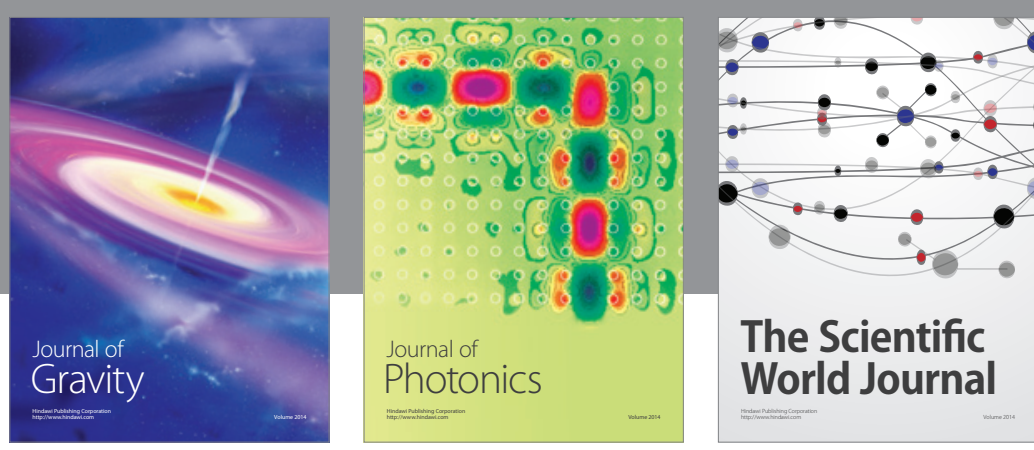

The Scientific World Journal
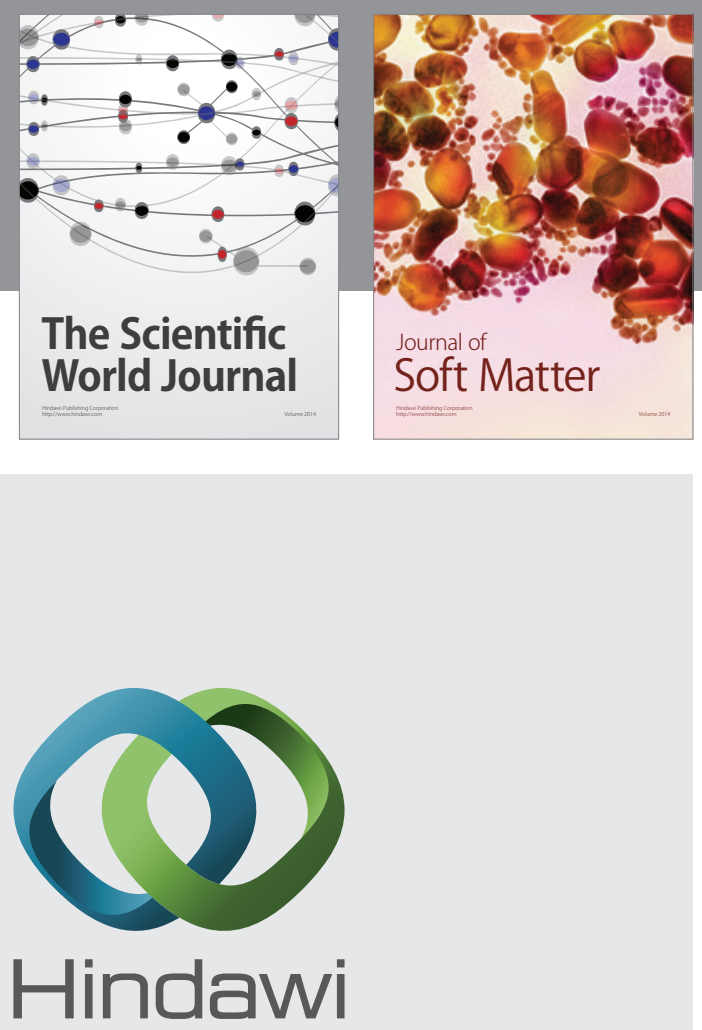

Submit your manuscripts at

http://www.hindawi.com

nternational Journal of

Statistical Mechanics
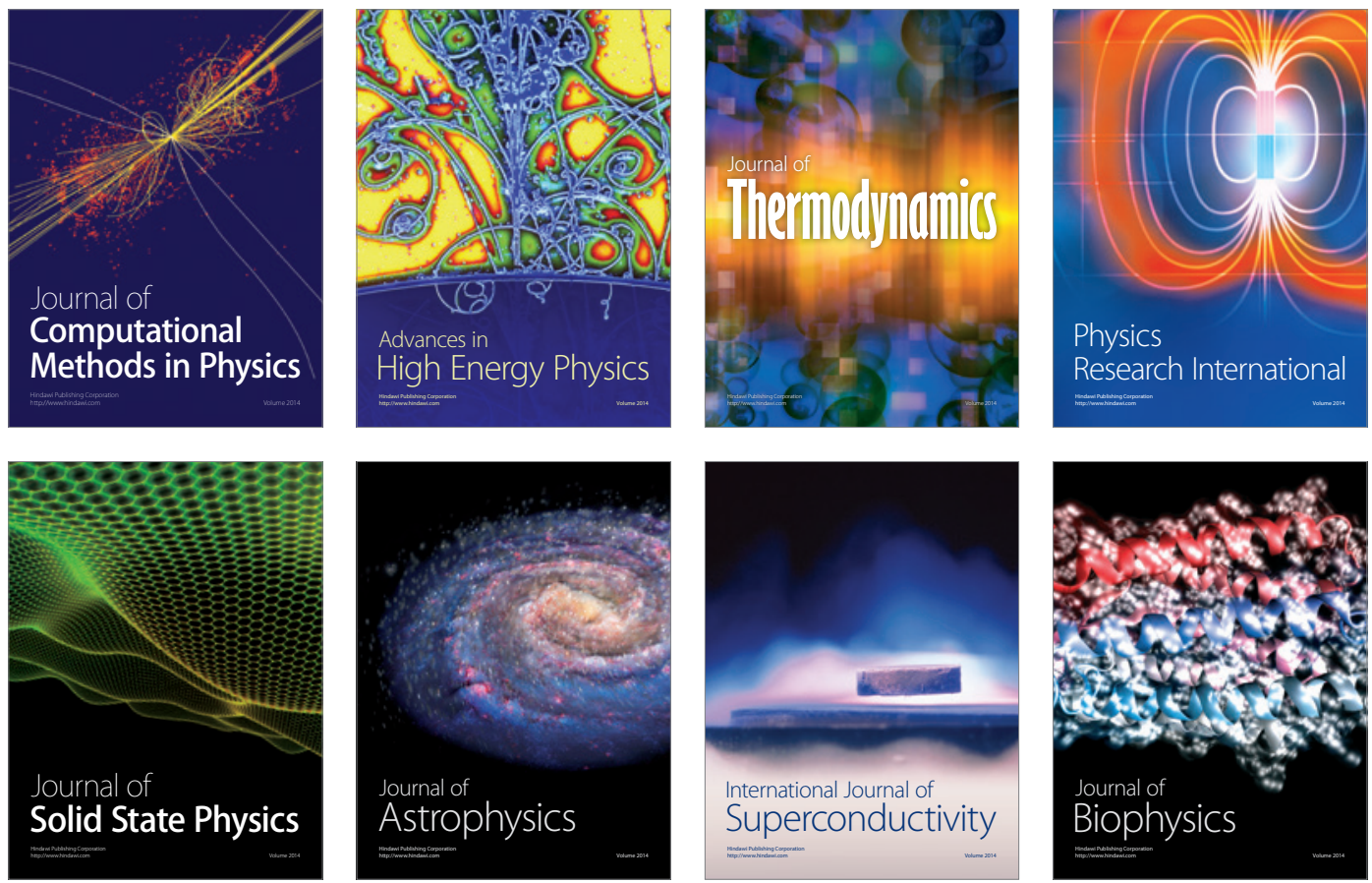
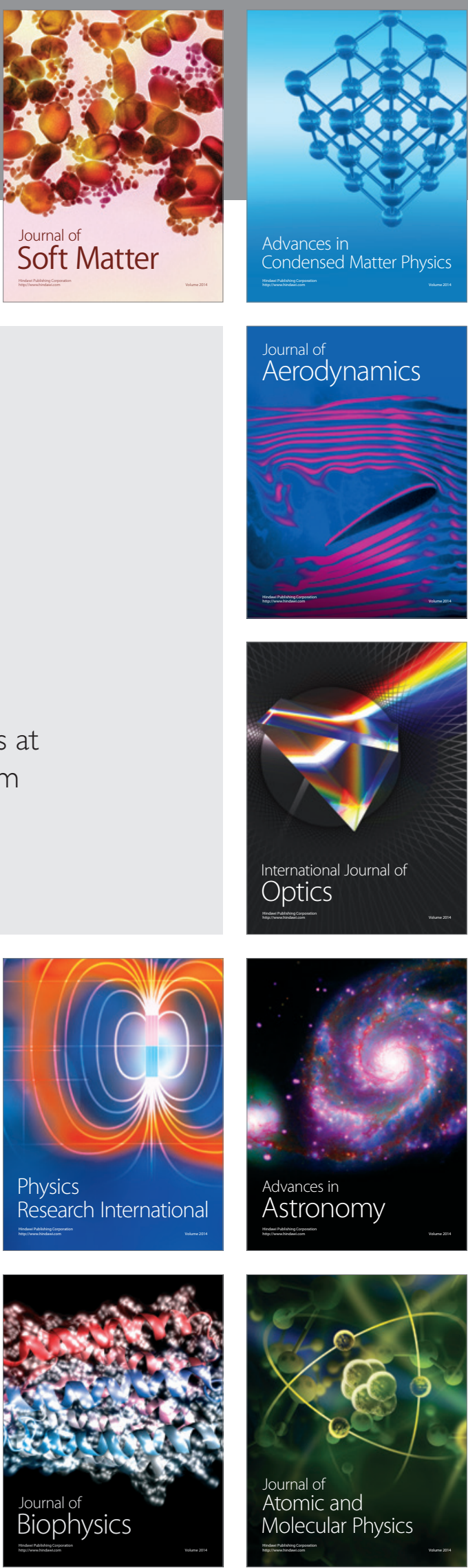